\title{
A Novel Image Segmentation Based on Clustering and Population-Based Optimisation
}

\author{
Seyed Jalaleddin Mousavirad ${ }^{1}$, Gerald Schaefer ${ }^{2}$, \\ Hossein Ebrahimpour-Komleh ${ }^{3}$, and Iakov Korovin ${ }^{4}$
}

1 Faculty of Engineering, Sabzevar University of New Technology, Sabzevar, Iran

2 Department of Computer Science, Loughborough University, Loughborough, UK

${ }^{3}$ Computer Engineering Department, University of Kashan, Kashan, Iran

4 Southern Federal University, Taganrog, Russia

\begin{abstract}
Image segmentation is an essential step in image processing and computer vision with many image segmentation algorithms having been proposed in the literature. Among these, clustering is one of the prominent approaches to achieve segmentation. Traditional clustering algorithms have been used extensively for this purpose, although they have disadvantages such as dependence on initialisation conditions and a tendency to find only local optima. To overcome these disadvantages, population-based metaheuristic algorithms can be applied.

In this paper, we propose a novel clustering algorithm based on human mental search (HMS) for image segmentation. HMS is a relatively new population-based metaheuristic inspired from the manner of searching in online auctions. HMS comprises three operators: mental search, which explores the neighbourhood of candidate solutions using Levy flight; grouping, which clusters candidate solutions; and moving candidate solutions towards a promising area. To verify the efficacy of the proposed algorithm, we conduct several experiments based on different criteria including mean cost function value, statistical analysis and image segmentation criteria. The obtained results confirm superior performance of our proposed algorithm compared to competitors.
\end{abstract}

Keywords: Image segmentation $\cdot$ Clustering $\cdot$ Optimisation • Population-based algorithms $\cdot$ Human mental search

\section{Introduction}

Image segmentation is a critical task in computer vision. It partitions an image in terms of pixel-based features into several homogeneous and disjoint regions so that the members within the same region share the same characteristics. Image segmentation is employed as a pre-processing step in many applications such as medical image processing $[4,15]$ or modelling of microstructures [16]. 
Clustering is one of the most commonly employed approaches for image segmentation. It divides an image into clusters (groups) so that the members located in the same cluster have more resemblance to each other than to those in others clusters. The $k$-means algorithm is the most popular clustering algorithm. It initialises $k$ cluster centres randomly and each pattern is then assigned to the closest cluster centre. In the next step, the location of each cluster centre is recomputed and the process continues until a stopping criterion is met. However, $k$-means suffers from some drawbacks such as dependence on initialisation and getting stuck in local optima.

Population-based metaheuristics are problem-independent optimisation algorithms with stochastic characteristics and can be used to overcome these drawbacks. They typically create a random population of candidate solutions which are then iteratively updated based on operations that are stochastic and share information within the population.

Popular population-based metaheuristics that have been used for clusteringbased image segmentation include particle swarm optimisation (PSO) [12,17], differential evolution (DE) [2,7], artificial bee colony (ABC) [13] and harmony search (HS) [20] among others.

Human mental search (HMS) [11] is a relatively new population-based metaheuristic that has shown competitive performance in solving optimisation problems [11]. HMS has three main operators: mental search, grouping, and movement. Mental search seeks around a candidate solution based on Levy flight, grouping clusters the population to find a promising region, and movement steers candidate solutions towards the promising area.

In this paper, we propose a novel image segmentation based on clustering and HMS. To this end, our encoding strategy is an array to define the cluster centres and we employ an objective function based on the mean squared error. An extensive set of experiments demonstrates very good segmentation performance and superiority over other methods.

The remainder of the paper is organised as follows. Section 2 summarises the human mental search algorithm, while Sect. 3 details our proposed image segmentation algorithm. Section 4 presents experimental results, and Sect. 5 concludes the paper.

\section{Human Mental Search}

Human mental search (HMS) [11] is a recent population-based metaheuristic algorithm where candidate solutions are bids in the space of online auctions.

The workings of HMS are detailed in Algorithm 1 in terms of pseudo-code. Like other population-based algorithms, HMS starts with a set of random candidate solutions. Candidate solutions try to move towards the optimum using mental search (to explore the vicinity of bids), grouping (to find the promising area), and movement (to steer bids toward the promising area) operators. In the following, we briefly explain these three operators. 


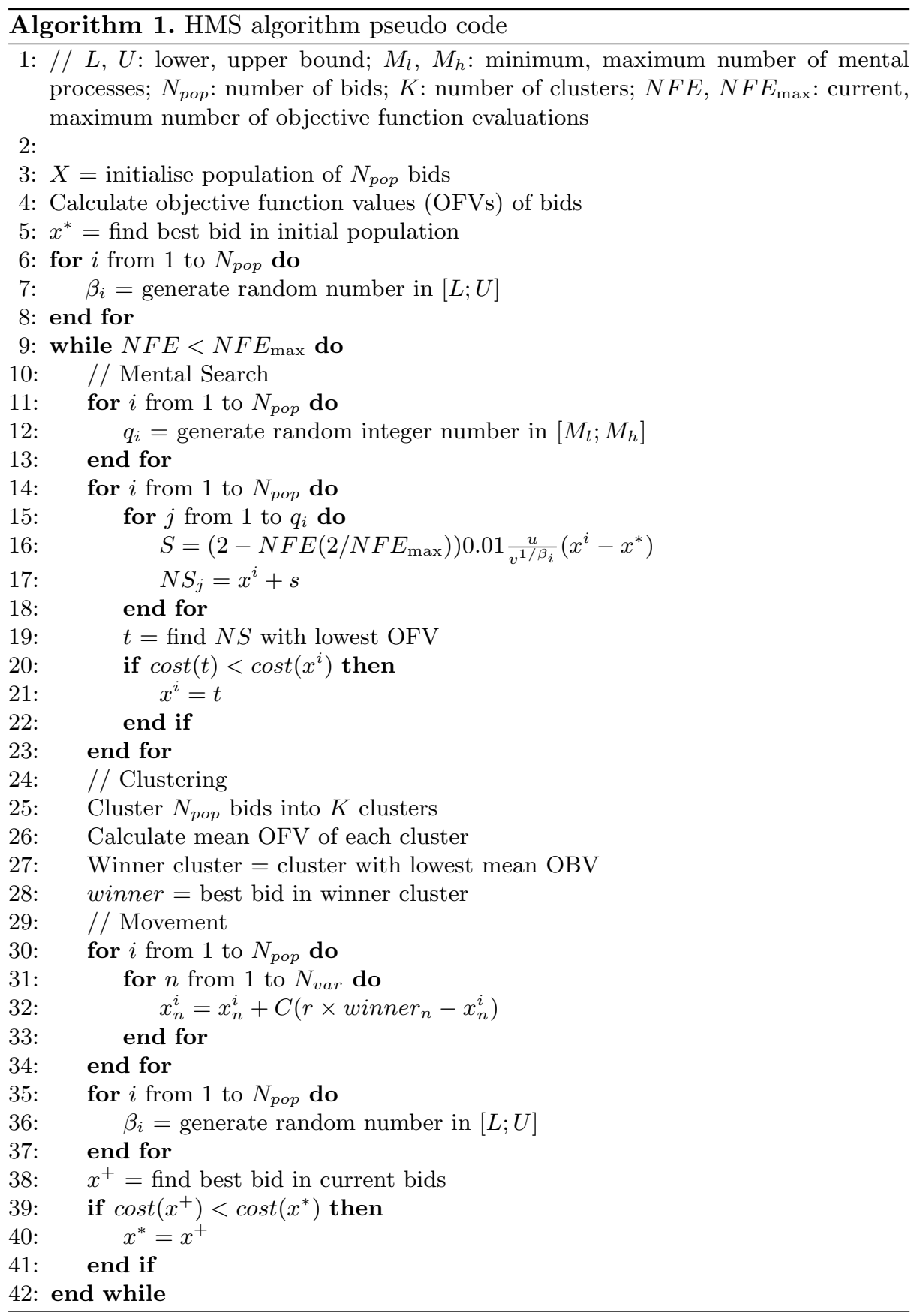




\subsection{Mental Search}

Here, each bid explores its vicinity based on a Levy flight distribution, resulting in both small and long jumps to enhance both exploration and exploitation ability of the algorithm simultaneously.

A bid $x^{i}$ is updated as

$$
x^{i}=x^{i}+S,
$$

with $S$ calculated as

$$
S=\left(2-N F E\left(2 / N F E_{\max }\right)\right) 0.01 \frac{u}{v^{1 / \beta}}\left(x^{i}-x^{*}\right),
$$

where $N F E$ is the number of objective function evaluations so far, $N F E_{\max }$ is the maximum number of function evaluations, $x^{*}$ is the best bid found so far, and $u$ and $v$ are two random numbers calculated as

$$
u \sim N\left(0, \sigma_{u}^{2}\right), v \sim N\left(0, \sigma_{v}^{2}\right)
$$

with

$$
\sigma_{u}=\left\{\frac{\Gamma(1+\beta) \sin \left(\frac{\pi \beta}{2}\right)}{\Gamma\left[\left(\frac{1+\beta}{2}\right)\right] \beta 2^{(\beta-1) / 2}}\right\}^{1 / \beta}, \sigma_{v}=1,
$$

where $\Gamma$ is a standard gamma function.

\subsection{Grouping}

A clustering approach is used to group the current population of bids. After clustering, which is performed using the $k$-means algorithm, similar bids are located in the same group. Then, the mean objective function value is calculated for each group, and the group with the lowest value selected as the winner group.

\subsection{Movement}

Here, bids move towards the best bid in the winner cluster as

$$
x_{n}^{t+1}=x_{n}^{t}+C\left(r \times \text { winner }_{n}^{t}-x_{n}^{t}\right),
$$

where $x_{n}^{t+1}$ is the $n$-th bid element at iteration $t+1$, winner ${ }_{n}^{t}$ is the $n$-th element of the best bid in the winner group, $t$ is the current iteration, $C$ is a constant, and $r$ is a number in $[0 ; 1]$ taken from a normal distribution.

\section{Proposed Segmentation Algorithm}

This paper introduces a novel clustering-based image segmentation algorithm based on clustering and HMS.

Assume that a dataset has $N$ objects, $O_{1}, O_{2}, \ldots, O_{N}$. A clustering algorithm tries to find $K$ cluster centres, $C_{1}, C_{2}, \ldots, C_{K}$ with the following conditions: 

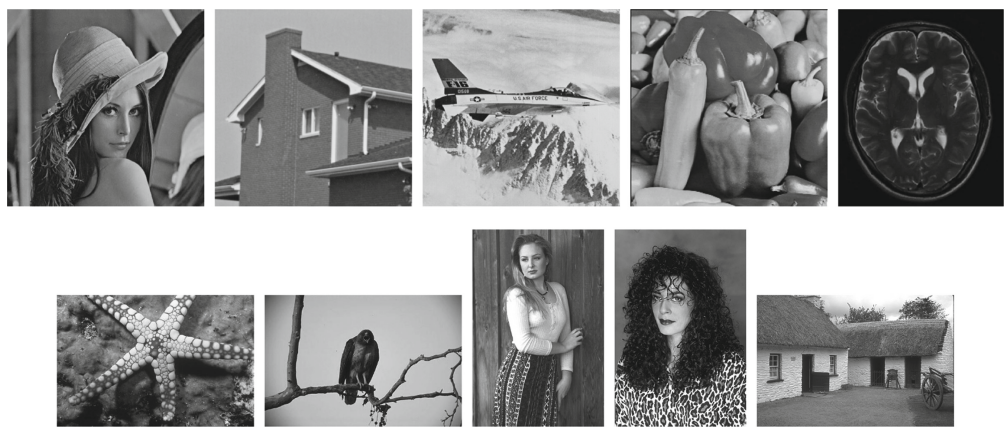

Fig. 1. Test image dataset.

1. Each cluster contains at least one object.

2. Distinct clusters have no objects in common.

3. The total number of cluster members is equal to the total number of objects in the dataset.

For our application of HMS, two issues need to be taken into account: encoding strategy and objective function. The encoding strategy determines the structure of each bid in HMS. We use an array to encode the cluster centres, and the length of the array is thus the number of cluster centres. Also, the upper and lower bound of the array is set to the maximum and minimum of pixel values in the image. As objective function, we use the mean squared error (MSE) defined as

$$
M S E=\frac{\sum_{j=1}^{K} \sum_{P_{i} \in C_{j}} d\left(P_{i}, C_{j}\right)}{n},
$$

where $d\left(P_{i}, c_{j}\right)$ is the Euclidean distance between pixel $P_{i}$ and its cluster centre $C_{j}$, and $n$ is the total number of pixels in the image.

Our image segmentation algorithm proceeds in the following steps:

Step 1: Parameter initialisation: population size $N_{\text {pop }}$, minimum $M_{l}$ and maximum $M_{h}$ number of mental searches, number of clusters for bid grouping $N$, number of objective function evaluations $N F E$, and number of clusters for image segmentation $K$.

Step 2: Generate an initial random set of bids.

Step 3: Calculate the objective function value for each bid using Eq. (6).

Step 4: Create a random integer number for each bid in $\left[M_{l} ; M_{h}\right]$ indicating the number of mental searches for the bid.

Step 5: Mental search: generate new bids based on Levy flight distribution in the vicinity of each bid.

Step 6: Replacement: in case a newly produced bid is better than the old one, it will replace the latter.

Step 7: Bid grouping: all bids in the search space are clustered using the $k$-means algorithm. 
Table 1. Parameter settings for all algorithms.

\begin{tabular}{l|l|c}
\hline Algorithm & Parameter & Value \\
\hline GA & Crossover probability & 0.8 \\
& Mutation probability & $1 /$ (number of thresholds) \\
\hline DE $[18]$ & Scaling factor & 0.5 \\
& Crossover probability & 0.1 \\
\hline PSO $[19]$ & Cognitive constant & 2 \\
& Social constant & 2 \\
& Inertia constant & 1 to 0 \\
\hline ABC $[5]$ & Limit & $n_{e} \times$ dimensionality of problem \\
\hline HS $[3]$ & Harmony memory considering rate & 0.9 \\
& Pitch adjusting rate & 0.1 \\
\hline HMS & Number of clusters & 5 \\
& $C$ & 2 \\
\hline
\end{tabular}

Step 8: Calculate the mean objective function value for each cluster.

Step 9: The cluster with the minimum mean objective function value is selected as the winner cluster.

Step 10: Other bids move towards the best bid in the winner cluster based on Eq. (5).

Step 11: Find the best bid in the current population and if it is better than the previous best bid, substitute the latter with it.

Step 12: If the stopping condition is not met, go back to Step 3.

\section{Experimental Results}

In our experiments, we select 10 images, shown in Fig. 1, which are commonly used in the literature: Lenna, House, Airplane, Peppers, and MRI, and five images from Berkeley image segmentation dataset [9], namely 12003, 42049, 181079,198054 , and 385028. We compare our algorithm with other populationbased algorithms that have been previously used for image segmentation.

The population size and the number of objective function evaluations for all algorithms are set to 50 and 6000, respectively, and all results are run for $K=5$. For HMS, we set the number of clusters used during grouping to 5 and $C$ to 2 , while $M_{l}=2, M_{h}=10, L=0.3$, and $U=1.99$. For the other algorithms, we adapt default values previously employed in this context, listed in Table 1. Since the algorithms are stochastic, we run each algorithm 25 times on each image, and report the average over these runs. We also rank the algorithms for each image and calculate Friedmann ranks which are obtained by averaging the ranks over the image set. 
Table 2. Objective function value results.

\begin{tabular}{|c|c|c|c|c|c|c|}
\hline Image & HMS & $\mathrm{ABC}$ & HS & PSO & $\mathrm{DE}$ & GA \\
\hline \multirow[t]{2}{*}{ Lenna } & 0.0453 & 0.0481 & 0.0464 & 0.0457 & 0.0475 & 0.0458 \\
\hline & 1 & 6 & 4 & 2 & 5 & 3 \\
\hline \multirow[t]{2}{*}{ Airplane } & 0.0335 & 0.0493 & 0.0351 & 0.0370 & 0.0336 & 0.0372 \\
\hline & 1 & 6 & 3 & 4 & 2 & 5 \\
\hline \multirow[t]{2}{*}{ House } & 0.0801 & 0.0873 & 0.0847 & 0.0796 & 0.0821 & 0.0803 \\
\hline & 2 & 6 & 5 & 1 & 4 & 3 \\
\hline \multirow[t]{2}{*}{ Peppers } & 0.1025 & 0.1084 & 0.1039 & 0.1024 & 0.1031 & 0.2538 \\
\hline & 2 & 5 & 4 & 1 & 3 & 6 \\
\hline \multirow[t]{2}{*}{ MRI } & 0.0217 & 0.0446 & 0.0234 & 0.0226 & 0.0218 & 0.0263 \\
\hline & 1 & 6 & 4 & 3 & 2 & 5 \\
\hline \multirow[t]{2}{*}{42049} & 0.0578 & 0.0793 & 0.0574 & 0.0665 & 0.0586 & 0.0593 \\
\hline & 2 & 6 & 1 & 5 & 3 & 4 \\
\hline \multirow[t]{2}{*}{12003} & 0.0606 & 0.0720 & 0.0618 & 0.0609 & 0.0606 & 0.0614 \\
\hline & 2 & 6 & 5 & 3 & 1 & 4 \\
\hline \multirow[t]{2}{*}{81079} & 0.0661 & 0.0812 & 0.0668 & 0.0745 & 0.0673 & 0.0677 \\
\hline & 1 & 6 & 2 & 5 & 3 & 4 \\
\hline \multirow[t]{2}{*}{198054} & 0.0738 & 0.0785 & 0.0745 & 0.0745 & 0.0739 & 0.0749 \\
\hline & 1 & 6 & 4 & 3 & 2 & 5 \\
\hline \multirow[t]{2}{*}{385028} & 0.0690 & 0.0752 & 0.0697 & 0.0693 & 0.0680 & 0.0699 \\
\hline & 2 & 6 & 4 & 3 & 1 & 5 \\
\hline Average rank & 1.5 & 5.9 & 3.6 & 3.0 & 2.6 & 4.4 \\
\hline Overall rank & 1 & 6 & 4 & 3 & 2 & 5 \\
\hline
\end{tabular}

Table 2 compares the results in terms of objective function values. As we can see, HMS is ranked first for 5 of the 10 images, and second for the other ones, leading to a clear best overall rank, followed by DE and PSO.

We also perform a statistical analysis, using the Wilcoxon signed rank test to compare our HMS-based algorithm with the other techniques. The null hypothesis $H_{0}$ here indicates no statistical difference between two algorithms, while the alternative hypothesis $H_{1}$ points to a statistically significant difference. The obtained $p$-values are 0.0020 for ABC, 0.0039 for HS, 0.0273 for PSO, 0.0488 for DE, and 0.0020 for GA and are thus all below 0.05 demonstrating statistical superiority of HMS.

We further assess our proposed algorithm using several image segmentation criteria, in particular:

- Borsotti criterion (BOR) [1]: an unsupervised criterion based on the number, the variance, and the area of segmented image regions. A lower BOR indicates a better segmentation performance. 
Table 3. BOR results.

\begin{tabular}{l|c|c|c|c|c|c}
\hline Image & HMS & ABC & HS & PSO & DE & GA \\
\hline Lenna & 0.0187 & 0.0279 & 0.0201 & 0.0285 & 0.0193 & 0.0245 \\
Airplane & 1 & 5 & 3 & 6 & 2 & 4 \\
& 0.0412 & 0.2650 & 0.3185 & 0.0609 & 0.2945 & 0.0778 \\
House & 1 & 4 & 6 & 2 & 5 & 3 \\
& 0.0220 & 0.0238 & 0.0236 & 0.0231 & 0.0230 & 0.0242 \\
Peppers & 1 & 5 & 4 & 3 & 2 & 6 \\
MRI & 0.0761 & 0.1072 & 0.1067 & 0.0924 & 0.1097 & 0.0894 \\
& 1 & 5 & 4 & 3 & 6 & 2 \\
42049 & 0.0538 & 0.1028 & 0.1063 & 0.1022 & 0.1002 & 0.0870 \\
& 1 & 5 & 6 & 4 & 3 & 2 \\
12003 & 0.1289 & 0.2387 & 0.3460 & 0.2175 & 0.2587 & 0.1669 \\
& 1 & 4 & 6 & 3 & 5 & 2 \\
181079 & 0.0375 & 0.1308 & 0.4101 & 0.0420 & 0.2839 & 0.0602 \\
& 1 & 4 & 6 & 2 & 5 & 3 \\
198054 & 0.1798 & 0.2870 & 0.3008 & 0.2840 & 0.3194 & 0.2678 \\
& 1 & 4 & 5 & 3 & 6 & 2 \\
385028 & 0.2449 & 0.4620 & 0.6141 & 0.1916 & 0.6244 & 0.3020 \\
& 2 & 4 & 5 & 1 & 6 & 3 \\
\hline Average rank & 0.1107 & 0.2108 & 0.2187 & 0.2060 & 0.2353 & 0.2136 \\
Overall rank & 1 & 3 & 5 & 2 & 6 & 4 \\
\hline
\end{tabular}

- Levine and Nazif interclass contrast (LNIC) [8]: an unsupervised criterion based on the sum of region contrasts weighted by their areas. A higher LNIC indicates a better segmentation performance.

- Levine and Nazif intra-class uniformity (LNIU) [8]: an unsupervised criterion based on the sum of normalised standard deviations of image areas. A higher LNIU indicates a better segmentation performance.

- Variation of information (VoI) [10]: a supervised criterion that calculates the information shared between a ground truth and a segmented image. A lower VoI indicates better segmentation performance.

- Probabilistic Rand index (PRI) [14]: a supervised criterion that computes the fraction of pairs of pixels whose labels are consistent between computed and ground truth segmentations. A higher PRI indicates better segmentation performance.

Since the latter two require a ground truth, we can only evaluate these measures on the images of the Berkeley dataset. 
Table 4. LNIC results.

\begin{tabular}{|c|c|c|c|c|c|c|}
\hline Image & HMS & $\mathrm{ABC}$ & HS & PSO & $\mathrm{DE}$ & GA \\
\hline \multirow[t]{2}{*}{ Lenna } & 0.2342 & 0.2321 & 0.2330 & 0.2364 & 0.2322 & 0.2307 \\
\hline & 2 & 5 & 3 & 1 & 4 & 6 \\
\hline \multirow[t]{2}{*}{ Airplane } & 0.1177 & 0.1070 & 0.1057 & 0.1084 & 0.1178 & 0.1160 \\
\hline & 2 & 5 & 6 & 4 & 1 & 3 \\
\hline \multirow[t]{2}{*}{ House } & 0.1760 & 0.1727 & 0.1737 & 0.1743 & 0.1732 & 0.1720 \\
\hline & 1 & 5 & 3 & 2 & 4 & 6 \\
\hline \multirow[t]{2}{*}{ Peppers } & 0.2326 & 0.2043 & 0.2232 & 0.2308 & 0.2302 & 0.2057 \\
\hline & 1 & 6 & 4 & 2 & 3 & 5 \\
\hline \multirow[t]{2}{*}{ MRI } & 0.7145 & 0.5265 & 0.7119 & 0.7007 & 0.7061 & 0.5849 \\
\hline & 1 & 6 & 2 & 4 & 3 & 5 \\
\hline \multirow[t]{2}{*}{42049} & 0.3976 & 0.2719 & 0.3429 & 0.3870 & 0.3421 & 0.3100 \\
\hline & 1 & 6 & 3 & 2 & 4 & 5 \\
\hline \multirow[t]{2}{*}{12003} & 0.2024 & 0.1957 & 0.1213 & 0.2018 & 0.1604 & 0.2192 \\
\hline & 2 & 4 & 6 & 3 & 5 & 1 \\
\hline \multirow[t]{2}{*}{181079} & 0.3095 & 0.2541 & 0.3225 & 0.3079 & 0.3008 & 0.3021 \\
\hline & 2 & 6 & 1 & 3 & 5 & 4 \\
\hline \multirow[t]{2}{*}{198054} & 0.6791 & 0.4541 & 0.6773 & 0.5106 & 0.6159 & 0.5612 \\
\hline & 1 & 6 & 2 & 5 & 3 & 4 \\
\hline \multirow[t]{2}{*}{385028} & 0.2638 & 0.2165 & 0.2657 & 0.2772 & 0.2573 & 0.2507 \\
\hline & 3 & 6 & 2 & 1 & 4 & 5 \\
\hline Average rank & 1.6 & 5.5 & 3.2 & 2.7 & 3.6 & 4.4 \\
\hline Overall rank & 1 & 6 & 3 & 2 & 4 & 5 \\
\hline Origir & im & $\mathrm{m}$ & & & & \\
\hline Segme & & seg & & Segmer & tation 5 & \\
\hline & GA & & $\mathrm{E}$ & PS & & \\
\hline & $\mathrm{HS}$ & & & & & \\
\hline
\end{tabular}

Fig. 2. Segmented images for image 385028 . 
Table 5. LNIU results.

\begin{tabular}{l|c|c|c|c|c|c}
\hline Image & HMS & ABC & HS & PSO & DE & GA \\
\hline Lenna & 0.0304 & 0.0301 & 0.0307 & 0.0302 & 0.0305 & 0.0303 \\
Airplane & 3 & 6 & 1 & 5 & 2 & 4 \\
& 0.1251 & 0.0620 & 0.1044 & 0.0698 & 0.1160 & 0.1733 \\
House & 2 & 6 & 4 & 5 & 3 & 1 \\
& 0.0283 & 0.0278 & 0.0280 & 0.0281 & 0.0280 & 0.0288 \\
Peppers & 2 & 6 & 4 & 3 & 5 & 1 \\
MRI & 0.0740 & 0.0626 & 0.0741 & 0.0689 & 0.0755 & 0.0658 \\
& 3 & 6 & 2 & 4 & 1 & 5 \\
42049 & 0.0648 & 0.0623 & 0.0646 & 0.0642 & 0.0643 & 0.0634 \\
& 1 & 6 & 2 & 4 & 3 & 5 \\
12003 & 0.1216 & 0.1166 & 0.1063 & 0.1163 & 0.1068 & 0.1048 \\
& 1 & 2 & 5 & 3 & 4 & 6 \\
181079 & 0.1055 & 0.1042 & 0.1006 & 0.1022 & 0.1006 & 0.0793 \\
& 1 & 2 & 4 & 3 & 5 & 6 \\
198054 & 0.1350 & 0.1190 & 0.1313 & 0.1317 & 0.1302 & 0.1304 \\
& 1 & 6 & 3 & 2 & 5 & 4 \\
385028 & 0.2123 & 0.1653 & 0.2004 & 0.1351 & 0.2422 & 0.1812 \\
& 2 & 5 & 3 & 6 & 1 & 4 \\
\hline Average rank & 1.8 & 4.6 & 3.4 & 3.8 & 3.4 & 4 \\
Overall rank & 1 & 6 & 2.5 & 4 & 2.5 & 5 \\
\hline
\end{tabular}

The BOR results in Table 3 show that HMS performs best for all but one image and is thus clearly ranked first overall with PSO coming second.

For LNIC, reported in Table 4, HMS yields the first rank for 5 images and second for a further 4, while similar results can be seen for LNIU in Table 5. For both criteria, our approach is the overall top-ranked algorithm.

Table 6 indicates the VoI results. Here, HMS obtained the first rank for 2 and second rank for the remaining 3 images, while GA, HS, and PSO rank first each for one image. Overall, HMS ranks first, followed by PSO and DE.

Table 7 lists the PRI results. For all images, HMS ranks first or second, resulting in an overall first rank.

Last not least, we show an example of the obtained results in Fig. 2 which also demonstrates the superiority of HMS.

Overall, it is clear that our proposed HMS approach outperforms all other algorithms based on all employed measures. 
Table 6. VoI results.

\begin{tabular}{l|c|c|c|c|c|c}
\hline Image & HMS & ABC & HS & PSO & DE & GA \\
\hline 42049 & 3.2839 & 3.3877 & 3.5316 & 3.2966 & 3.3127 & 3.2037 \\
& 2 & 5 & 6 & 3 & 4 & 1 \\
12003 & 3.1758 & 3.6519 & 2.7900 & 3.2028 & 3.2215 & 3.4100 \\
181079 & 2 & 6 & 1 & 3 & 4 & 5 \\
& 3.0395 & 3.4102 & 3.1569 & 3.1781 & 3.0423 & 3.0594 \\
198054 & 1 & 6 & 4 & 5 & 2 & 3 \\
& 2.2528 & 2.5951 & 2.2880 & 2.2952 & 2.2729 & 2.3517 \\
385028 & 1 & 6 & 3 & 4 & 2 & 5 \\
& 3.8173 & 3.9470 & 3.8233 & 3.7099 & 3.8688 & 3.8769 \\
& 2 & 6 & 3 & 1 & 4 & 5 \\
\hline Average rank & 1.6 & 5.8 & 3.4 & 3.2 & 3.2 & 3.8 \\
Overall rank & 1 & 6 & 4 & 2.5 & 2.5 & 5 \\
\hline
\end{tabular}

Table 7. PRI results.

\begin{tabular}{l|c|c|c|c|c|c}
\hline Image & HMS & ABC & HS & PSO & DE & GA \\
\hline 42049 & 0.6110 & 0.6092 & 0.4331 & 0.6088 & 0.4836 & 0.6002 \\
& 1 & 2 & 6 & 3 & 5 & 4 \\
12003 & 0.4658 & 0.4032 & 0.3894 & 0.4017 & 0.4461 & 0.5692 \\
& 2 & 4 & 6 & 5 & 3 & 1 \\
181079 & 0.4885 & 0.4653 & 0.4800 & 0.4767 & 0.470 & 0.4889 \\
& 2 & 6 & 3 & 4 & 5 & 1 \\
198054 & 0.4824 & 0.6426 & 0.3498 & 0.4730 & 0.3434 & 0.4015 \\
& 2 & 1 & 5 & 3 & 6 & 4 \\
385028 & 0.5386 & 0.5059 & 0.5267 & 0.5333 & 0.4907 & 0.5240 \\
& 1 & 5 & 3 & 2 & 6 & 4 \\
\hline Average rank & 1.6 & 3.6 & 4.6 & 3.4 & 5 & 2.8 \\
Overall rank & 1 & 4 & 5 & 3 & 6 & 2 \\
\hline
\end{tabular}

\section{Conclusions}

This paper proposes a novel clustering-based image segmentation based on human mental search (HMS). Our proposed algorithm encodes cluster centres as the candidate solutions, uses the mean squared error as objective function and employs HMS for optimisation of the latter. The proposed algorithm is compared with five other metaheuristic algorithms and the obtained experimental results verify the efficacy of the proposed algorithm for image segmentation and show it to outperform the other approaches. In future, we plan to extend our approach to find the number of optimal clusters automatically, while integrating 
a local search mechanism is also being investigated. We also currently explore our algorithm in the context of video analysis [6].

Acknowledgements. This paper is published due to the financial support of the Federal Target Programme of the Ministry of Science and Higher Education of the Russian Federation, project unique identifier RFMEFI60819X0281.

\section{References}

1. Borsotti, M., Campadelli, P., Schettini, R.: Quantitative evaluation of color image segmentation results. Pattern Recogn. Lett. 19(8), 741-747 (1998)

2. Das, S., Konar, A.: Automatic image pixel clustering with an improved differential evolution. Appl. Soft Comput. 9(1), 226-236 (2009)

3. Geem, Z.W., Kim, J.H., Loganathan, G.V.: A new heuristic optimization algorithm: harmony search. Simulation 76(2), 60-68 (2001)

4. Jamil, U., Sajid, A., Hussain, M., Aldabbas, O., Alam, A., Shafiq, M.U.: Melanoma segmentation using bio-medical image analysis for smarter mobile healthcare. J. Ambient Intell. Hum. Comput. 10, 1-22 (2019)

5. Karaboga, D., Akay, B.: A comparative study of artificial bee colony algorithm. Appl. Math. Comput. 214(1), 108-132 (2009)

6. Korovin, I.S., Khisamutdinov, M.V.: Obtaining a noise-free image based on an analysis of an unstabilized video sequence under conditions of a probable optical flow failure. In: 12th International Conference on Machine Vision (2019)

7. Kwedlo, W.: A clustering method combining differential evolution with the $k$-means algorithm. Pattern Recogn. Lett. 32(12), 1613-1621 (2011)

8. Levine, M.D., Nazif, A.M.: Dynamic measurement of computer generated image segmentations. IEEE Trans. Pattern Anal. Mach. Intell. 7(2), 155-164 (1985)

9. Martin, D., Fowlkes, C., Tal, D., Malik, J.: A database of human segmented natural images and its application to evaluating segmentation algorithms and measuring ecological statistics. In: 8th International Conference on Computer Vision, vol. 2, pp. 416-423 (2001)

10. Meilă, M.: Comparing clusterings: an axiomatic view. In: 22nd International Conference on Machine Learning, pp. 577-584 (2005)

11. Mousavirad, S.J., Ebrahimpour-Komleh, H.: Human mental search: a new population-based metaheuristic optimization algorithm. Appl. Intell. 47(3), 850887 (2017). https://doi.org/10.1007/s10489-017-0903-6

12. Omran, M.G., Engelbrecht, A.P., Salman, A.: Image classification using particle swarm optimization. In: Recent Advances in Simulated Evolution and Learning, pp. 347-365. World Scientific (2004)

13. Ozturk, C., Hancer, E., Karaboga, D.: Improved clustering criterion for image clustering with artificial bee colony algorithm. Pattern Anal. Appl. 18(3), 587-599 (2014). https://doi.org/10.1007/s10044-014-0365-y

14. Pantofaru, C., Hebert, M.: A comparison of image segmentation algorithms. Technical report, Robotics Institute (2005)

15. Rose, R.A., Annadhason, A.: GHT based automatic kidney image segmentation using modified AAM and GBDT. Health Technol. 10, 1-10 (2019)

16. Sanei, S.H.R., Fertig III, R.S.: Uncorrelated volume element for stochastic modeling of microstructures based on local fiber volume fraction variation. Compos. Sci. Technol. 117, 191-198 (2015) 
17. Shi, Y., Eberhart, R.: A modified particle swarm optimizer. In: IEEE International Conference on Evolutionary Computation, pp. 69-73 (1998)

18. Storn, R., Price, K.: Differential evolution-a simple and efficient heuristic for global optimization over continuous spaces. J. Global Optim. 11(4), 341-359 (1997)

19. Suganthan, P.N., et al.: Problem definitions and evaluation criteria for the CEC 2005 special session on real-parameter optimization. Technical report, Nanyang Technological University Singapore (2005)

20. Wang, L., Yufeng, Y., Liu, J.: Clustering with a novel global harmony search algorithm for image segmentation. Int. J. Hybrid Inf. Technol. 9(2), 183-194 (2016) 